\title{
Utilidad de las redes neuronales artificiales en la predicción de cáncer de próstata en la biopsia transrectal
}

\author{
A. Rodríguez Alonso, S. Pértega Díaz*, A. González Blanco, S. Pita Fernández*, \\ G. Suárez Pascual, M.A. Cuerpo Pérez \\ Servicio de Urología, Hospital Arquitecto Marcide - Profesor Novoa Santos. Ferrol. La Coruña. \\ *Unidad de Epidemiología Clínica y Bioestadística. Hospital Juan Canalejo. La Coruña.
}

Actas Urol Esp 2006; 30 (1): 18-24

\section{RESUMEN}

\section{UTILIDAD DE LAS REDES NEURONALES ARTIFICIALES EN LA PREDICCIÓN DE CÁNCER DE PRÓSTATA EN LA BIOPSIA TRANSRECTAL}

Objetivo: Determinar si el desarrollo de una red neuronal artificial (RNA) formada por variables clínicas permite predecir el resultado de la biopsia prostática (BP).

Material y métodos: Pacientes ( $\mathrm{n}=953$ ) sometidos a BP en el Hospital Arquitecto Marcide, Ferrol, entre enero-2000 y junio-2005. Las variables estudiadas fueron edad, PSA, tacto rectal y volumen prostático, disponiendo de todos estos datos en 843 casos. Para determinar factores relacionados con el diagnóstico de cáncer de próstata (CP), se desarrollaron un análisis de regresión logística y una red neuronal "feed-forward", con tres nodos en su capa oculta y un nodo de salida, que representa la probabilidad de CP. Ambos modelos fueron construidos a partir de una muestra aleatoria de $\mathrm{n}=643$ pacientes (set de derivación). La capacidad predictiva de ambos modelos se valoró con los 200 pacientes restantes (set de validación), mediante curvas ROC y su área bajo la curva (ABC).

Resultados: Se detectó CP en 500 (59,3\%) casos. Ajustando por edad, PSA, tacto rectal y volumen prostático, en un modelo de regresión logística multivariante, se observó que todas las variables predecían CP de forma independiente. Las ABC fueron de 0,693 para el PSA, 0,707 para el volumen prostático, 0,815 para la regresión logística y 0,819 para la RNA. La capacidad predictiva de la RNA fue significativamente superior a la del PSA ( $\mathrm{p}=0,002)$ y volumen prostático $(\mathrm{p}<0,001)$ y similar a la de la regresión logística $(\mathrm{p}=0,760)$.

Conclusiones: La RNA presenta una capacidad de predicción de CP significativamente superior a los métodos diagnósticos unimodales, y similar a la regresión logística.

Palabras clave: Próstata. Biopsia. Neoplasia prostática. Red neuronal artificial. Regresión logística. Diagnóstico.

\section{ABSTRACT \\ THE UTILITY OF ARTIFICIAL NEURAL NETWORKS IN THE PREDICTION OF PROSTATE CANCER ON TRANSRECTAL BIOPSY}

Objective: To determine whether the development of an artificial neural network (ANN) made up of clinical variables allows for the prediction of prostate biopsy (PB) outcome.

Materials and methods: Patients ( $\mathrm{n}=953)$ underwent PB at the Arquitecto Marcide Hospital in Ferrol (Spain), between january 2000 and june 2005. The variables studied were age, PSA, digital rectal examination (DRE) and prostate volu$\mathrm{me}$, data for all of which were available in 843 cases. In order to determine factors related to prostate cancer (PC) diagnosis, a logistic regression analysis and a feed-forward neural network were developed, including three hidden layer nodes and an output node, representing the probability of PC. Both models were constructed from a random sample of $\mathrm{n}=643$ patients (derivation set). The predictive capacity was assessed with the remaining 200 patients (validation set), by means of ROC curves and the area under the curve (AUC).

Results: PC was detected in 500 (59.3\%) cases. Adjusting for age, PSA, digital rectal examination and prostate volume, in a multivariate logistic regression model it was observed that all the variables were independent predictors of PC. The AUC were 0.693 for PSA, 0.707 for prostate volume, 0.815 for logistic regression and 0.819 for ANN. The predictive capacity of the ANN was significantly higher than that of the PSA $(p=0.002)$ and prostate volume $(p<0,001)$ and similar to that of logistic regression ( $\mathrm{p}=0.760)$.

Conclusions: The ANN shows a PC prediction capacity that is significantly higher than unimodal diagnosis methods, and similar to that of logistic regression.

Keywords: Prostate. Biopsy. Prostatic neoplasms. Neural networks. Logistic regression. Diagnosis.

Cuadro de abreviaturas: CP: Cáncer de próstata. BP: Biopsia de próstata. RNA: Red neuronal artificial. PSA: Antígeno prostático específico. Ln: Logaritmo neperiano. ROC: Receiver Operating Characteristic. ABC: Área bajo la curva. 95\%IC: 95\% intervalo de confianza. 
$\mathrm{E}_{\mathrm{r}}^{1}$ diagnóstico del cáncer de próstata $(\mathrm{CP})$ se realiza mediante biopsia prostática (BP), fundamentándose las indicaciones de esta prueba en las características del tacto rectal y la cifra de PSA.

La sensibilidad del tacto rectal en el diagnóstico del $\mathrm{CP}$ se reduce progresivamente, de forma paralela al incremento del número de pacientes diagnosticados en estadio T1c, que en la actualidad representan aproximadamente el 50\% de todos $\operatorname{los} \operatorname{casos}^{1}$. La utilización del PSA permite aumentar la detección de CP en un $81 \%$, con respecto al uso aislado del tacto rectal ${ }^{2}$.

El PSA es considerado de forma generalizada como el mejor test para realizar el diagnóstico precoz del $\mathrm{CP}^{3,4}$. A pesar de ello, el PSA presenta una baja especificidad, ya que sólo un $25 \%$ de los pacientes con PSA 4-10 ng/ml presentarán un $\mathrm{CP}$ en la $\mathrm{BP}^{2,5}$. La sensibilidad del PSA tampoco se sitúa a un nivel óptimo, ya que se estima que el 20\% de los pacientes con CP tienen un PSA por debajo de $4 \mathrm{ng} / \mathrm{ml}^{6,7}$.

Estas limitaciones son fundamentalmente debidas a que el PSA es una molécula órganoespecifica, pero no cáncer-específica, por lo que su elevación no pronostica necesariamente un $\mathrm{CP}$, siendo por tanto imprescindible realizar una $\mathrm{BP}$ para establecer el diagnóstico de $\mathrm{CP}^{3,6,8}$.

Esta problemática ha estimulado la utilización de diferentes métodos estadísticos, que combinando parámetros clínicos como PSA, tacto rectal y edad, permiten obtener una información más personalizada y facilitar la toma de decisiones.

Las técnicas estadísticas utilizadas para la predicción del resultado de una $\mathrm{BP}$, a partir de datos clínicos, se han centrado habitualmente en el uso de modelos de regresión logística multivariante. Sin embargo, en los últimos años se ha planteado la posibilidad de que la complejidad de este tipo de datos pueda exceder la capacidad predictiva de los métodos estadísticos habituales, señalando a las redes neuronales artificiales (RNA) como una interesante herramienta alternativa $^{9}$.

Una red neuronal artificial es un modelo matemático no lineal, de naturaleza computacional, con una arquitectura inspirada en la organización neuronal biológica ${ }^{10}$. Frente a los métodos estadísticos estándar, no requiere de ningún conocimiento previo acerca del fenómeno de estudio, ni hipótesis sobre su distribución, modelizando de modo natural cualquier tipo de relación no lineal con la variable respuesta, así como la existencia de posibles interacciones entre las variables explicativas. Dicha flexibilidad ha incrementado su popularidad en los últimos años, con aplicaciones a distintos problemas en la investigación biomédica ${ }^{11}$. En particular, en el campo de la Urologia, han sido utilizadas con éxito para la predicción del estadio patológico del $\mathrm{CP}^{12}$, en la predicción de la progresión tras prostatectomía radical $^{13}$ y en el diagnóstico de $\mathrm{CP}$ en la biopsia ${ }^{7}$. No obstante, mientras algunos autores señalan su mayor capacidad predictiva frente a los modelos de regresión habituales ${ }^{14}$, otros no encuentran diferencias ${ }^{12}$.

El objetivo de este estudio es determinar si el desarrollo de una RNA formada por variables clínicas permite predecir la existencia de $\mathrm{CP}$ en la biopsia prostática.

\section{MATERIAL Y MÉTODOS}

Se realizó un estudio observacional de prevalencia incluyendo 953 pacientes a los que se les practicó de forma consecutiva una $\mathrm{BP}$ en el Servicio de Urología del Complejo Hospitalario Arquitecto Marcide-Profesor Novoa Santos, de Ferrol, entre enero de 2000 y junio de 2005.

De todos ellos se estudió su edad, PSA total, tacto rectal y volumen prostático determinado por ecografía transrectal. El número final de pacientes en los que se disponía de todas las variables fue de 843 .

Los criterios generales de biopsia fueron: tacto rectal sospechoso, PSA >10ng/ml, PSA entre 4$10 \mathrm{ng} / \mathrm{ml}$, si cociente PSA libre/total $<25 \%$ y/o PSA densidad $>0,15$.

La BP fue realizada mediante abordaje transrectal. Hasta diciembre de 2001, la BP fue de 6 cilindros, y de 10 cilindros a partir de esta fecha. Desde diciembre de 2002, se realizó BP ampliada de 24 cilindros a aquellos pacientes de edad $\leq 70$ años, con una o más BP previas negativas o bien, edad $\leq 75$ años, con dos o más BP previas negativas.

La determinación del PSA se efectuó mediante el ensayo ADVIA Centaur ${ }^{\circledR}$ (Bayer Diagnostics, Tarrytown, New York). 
La ecografia transrectal fue realizada utilizando un ecógrafo BK Leopard 2001, con un transductor transrectal biplanar de 7,5 Mhz y una aguja de biopsia de 18G.

El volumen prostático fue calculado mediante ecografía transrectal (TRUS), aplicando la fórmula del volumen de la elipse: diámetro cráneo-caudal $\mathrm{x}$ ántero-posterior $\mathrm{x}$ transversal $\mathrm{x} \pi / 6^{15}$.

Análisis estadístico: Se realizó un análisis descriptivo de las variables estudiadas. La comparación de variables cuantitativas entre grupos se realizó mediante el test $\mathrm{t}$-Student o el test de Mann-Whitney, según procediese. El contraste de normalidad se realizó con el test de KolmogorovSmirnov. Para las variables cualitativas se empleó el estadístico $\chi^{2}$ y el cálculo del OR con su 95\% IC. Para determinar factores relacionados con el diagnóstico de $\mathrm{CP}$ se desarrollaron paralelamente un análisis de regresión logística y una red neuronal "feed-forward" (o perceptrón multicapa). Para el modelo de regresión logística, se siguió una estrategia de pasos sucesivos, incluyendo los términos de interacción que resultaron estadísticamente significativos. Se demostró una relación lineal entre las variables estudiadas y la probabilidad de CP, excepto en el PSA, por ello se utilizó el logaritmo neperiano de PSA (Ln PSA). A su vez, la red neuronal consta de tres nodos en su capa oculta y un único nodo de salida, que representa la probabilidad de cáncer de próstata en la biopsia (Fig. 1). Ambos modelos fueron cons-

truidos a partir de un set de derivación que incluía una muestra aleatoria de 643 pacientes. La capacidad predictiva de ambas técnicas se valoró en los 200 sujetos restantes (set de validación), mediante las correspondientes curvas ROC y su área bajo la curva ( $\mathrm{ABC}$ ). $\mathrm{El} \mathrm{ABC}$ fue comparado mediante el procedimiento descrito por DeLong et $\mathrm{al}^{16}$. $\mathrm{El}$ análisis estadístico se realizó con los programas SPSS 12.5 para Windows, EPIDAT 3.0 y Neural Connection 2.1 (SPSS).

\section{RESULTADOS}

Las características clínicas de los pacientes se recogen en la Tabla 1.

La cifra de PSA fue $\leq 4 \mathrm{ng} / \mathrm{ml}$ en $19(2,3 \%)$ pacientes, entre 4,1 y $10 \mathrm{ng} / \mathrm{ml}$ en $402(47,7 \%)$ individuos, entre 10,1 y $20 \mathrm{ng} / \mathrm{ml}$ en $239(28,3 \%)$ y superior a $20 \mathrm{ng} / \mathrm{ml}$ en $183(21,7 \%)$ casos.

Se estableció el diagnóstico de CP en 500 $(59,3 \%)$ casos. En los pacientes del set de derivación $(n=643)$, se detectó CP en $371(57,7 \%)$ casos y patología benigna en $272(42,3 \%)$.

El análisis de los resultados se llevó a cabo sólo con los pacientes del set de derivación, detectándose diferencias significativas entre los pacientes con patología benigna y $\mathrm{CP}$, que se detallan en la Tabla 2.

Tras ajustar por edad, tacto rectal, volumen prostático y Ln PSA, en un modelo de regresión logística multivariante, se demostró que todas estas variables predecían de forma independiente la probabilidad de detectar $\mathrm{CP}$ en la biopsia (Tabla 3).

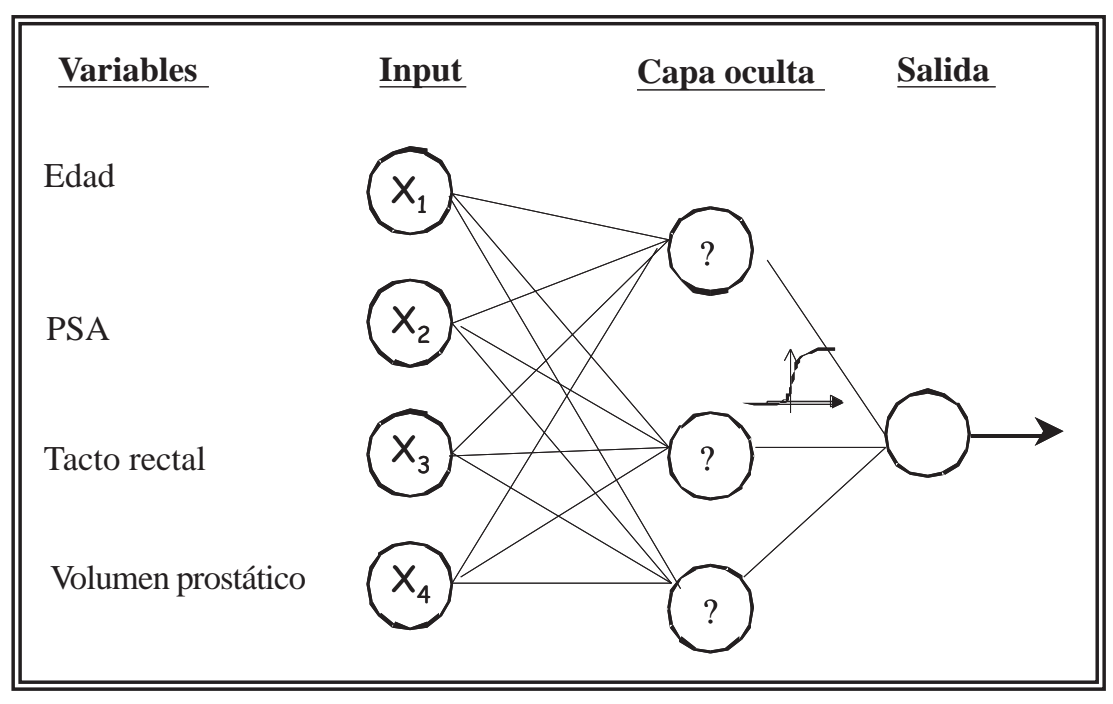

FIGURA 1: Estructura de la Red Neuronal Artificial construida para predecir cáncer de próstata, ajustando por diversas covariables. Set de derivación.
Las curvas ROC fueron realizadas con los pacientes del set de validación. Las áreas bajo la curva ROC fueron de 0,693 (95\%IC= $0,622 ; 0,765)$ para el PSA; 0,707 $(95 \% \mathrm{IC}=0,631 ; 0,783)$ para el volumen prostático; $0,815(95 \% \mathrm{IC}=$ $0,751 ; 0,880)$ para la regresión logistica y $0,819(95 \% \mathrm{IC}=0,754$; 0,884) para la red neuronal (Fig. 2). La capacidad predictiva de la red neuronal, determinada por el $A B C$, fue significativamente mayor que la del PSA $(\mathrm{p}=0,002)$ y la del volumen prostático $(\mathrm{p}<0,001)$. No se observaron diferencias signifi- 
Tabla 1

Características clínicas de los pacientes del estudio

\begin{tabular}{|c|c|c|c|c|c|c|}
\hline & & Media & DT & Mediana & Mínimo & Máximo \\
\hline $\operatorname{Edad}^{1}$ & & 70,1 & 7,4 & 70 & 48 & 91 \\
\hline Volumen prostático ${ }^{2}$ & & 56,5 & 29,4 & 49 & 7,40 & 194 \\
\hline \multirow[t]{2}{*}{$\mathrm{PSA}^{3}$} & & 25,5 & 91,0 & 10,1 & 0,30 & 1504,7 \\
\hline & & $\mathbf{n}$ & $\%$ & & & \\
\hline Tacto rectal & $\begin{array}{l}\text { No sospechoso } \\
\text { Sospechoso }\end{array}$ & $\begin{array}{l}467 \\
376\end{array}$ & $\begin{array}{l}55,4 \% \\
44,6 \%\end{array}$ & & & \\
\hline Diagnóstico de biopsia & $\begin{array}{l}\text { Negativo } \\
\text { Positivo }\end{array}$ & $\begin{array}{l}343 \\
500\end{array}$ & $\begin{array}{l}40,7 \% \\
59,3 \%\end{array}$ & & & \\
\hline
\end{tabular}

1 Años.

DT: Desviación típica

2 Centímetros cúbicos.

$3 \mathrm{ng} / \mathrm{ml}$.

Tabla 2

Diferencias observadas entre los pacientes sin y con cáncer de próstata, calculadas en los pacientes del set de derivación

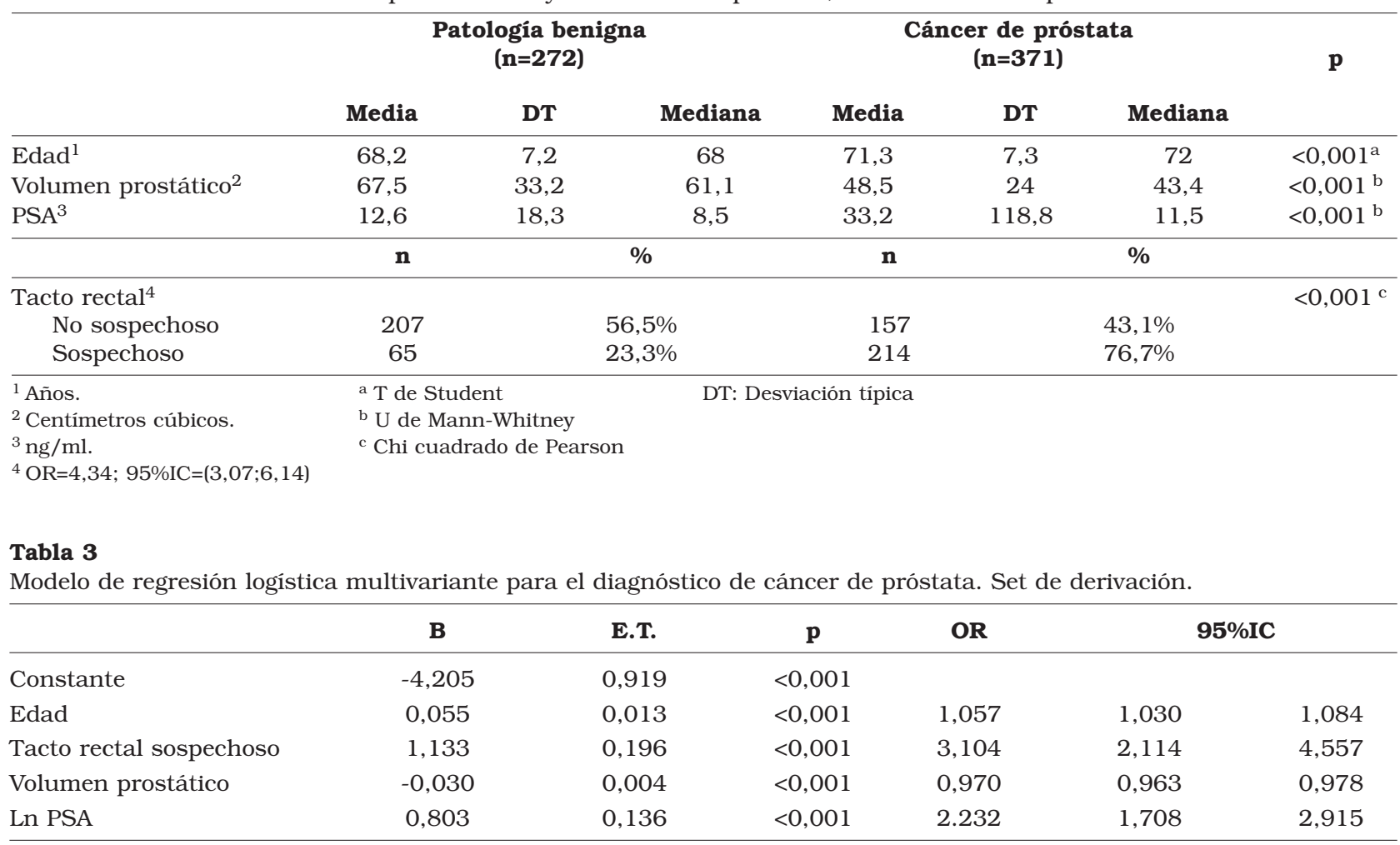

B: Coeficiente de regresión. ET: Error típico. Ln PSA: Logaritmo neperiano de PSA

OR: Odds ratio. 95\%IC: 95\% intervalo de confianza

cativas entre las $\mathrm{ABC}$ de la red neuronal y la regresión logística $(\mathrm{p}=0,760)$.

En la Tabla 4 se pueden observar el punto de corte y la especificidad para unos valores predeterminados de sensibilidad del 95\% y 90\%, así como el ABC del PSA, el volumen prostático, el modelo de regresión logística y la red neuronal.

\section{DISCUSIŌN}

La sospecha diagnóstica de CP se fundamenta en los hallazgos del tacto rectal, la edad, la historia familiar, la cifra de PSA y los parámetros derivados del $\mathrm{PSA}^{7}$.

Las dos indicaciones de biopsia clásicamente consideradas como absolutas son un tacto rectal 


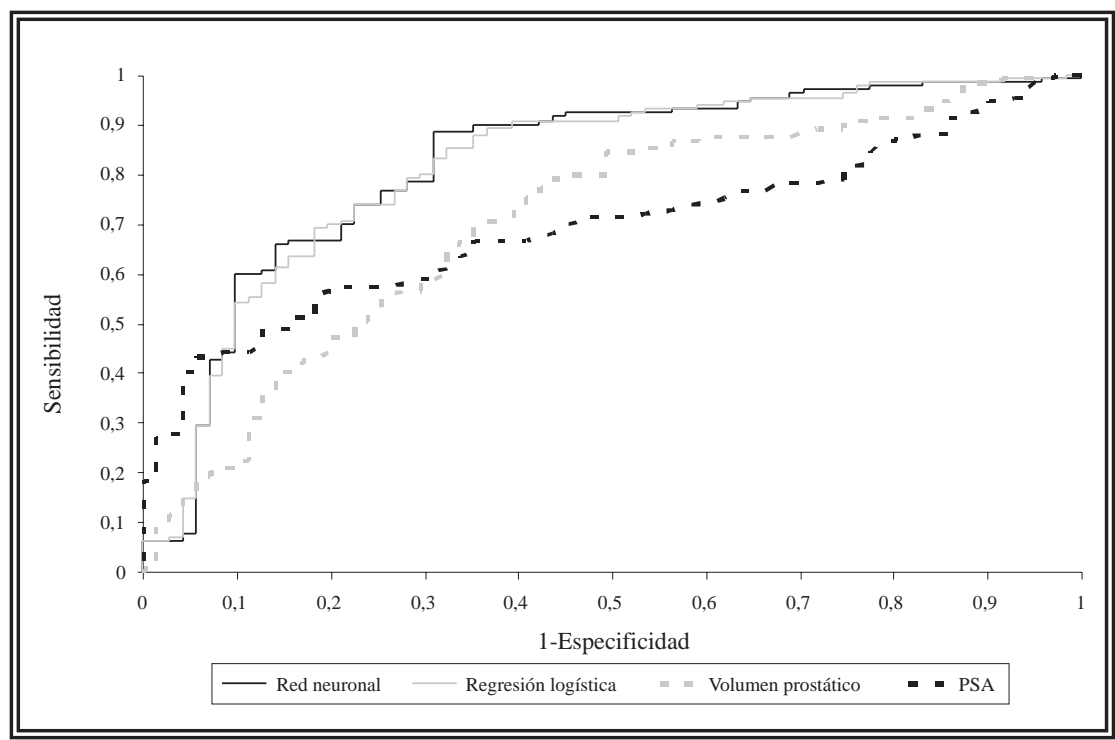

FIGURA 2: Curvas ROC de PSA (ABC=0,693), Volumen prostático (ABC=0,707), Regresión Logistica $(A B C=0,815)$ y Red Neuronal $(A B C=0,819)$.

sospechoso y/o un PSA >10 ng/ml. El intervalo de PSA 4-10 $\mathrm{ng} / \mathrm{ml}$ es considerado como una "zona gris" desde el punto de vista diagnóstico, por el solapamiento entre el PSA producido por los tejidos hiperplásico y tumoral, que da lugar a una importante reducción de la especificidad del test ${ }^{17,18}$. Para intentar incrementar la especificidad del PSA y reducir, por lo tanto, el número de biopsias innecesarias, se han utilizado diversas estrategias basadas en el PSA: cociente PSA libre/total, densidad de PSA, velocidad de PSA y PSA ajustado por edad ${ }^{2,6}$.

Sin embargo, no es sencillo predecir el resultado de una BP basándose en estas variables, tanto si se analizan de forma unimodal, o combinadas entre sí mediante métodos estadísticos convencionales, ya que dichas variables no siempre presentan una relación lineal con el CP. Las RNA representan, a priori, una solución para este problema, ya que combinan diversos parámetros con capacidad demostrada para predecir CP, buscando entre ellos relaciones no lineales, que los métodos estadísticos convencionales no permiten detectar ${ }^{19}$. Para modelizar la relación de las características clínicas estudiadas con el resultado de la BP, se optó por una red neuronal de tipo perceptrón multicapa, que es la red utilizada con mayor frecuencia en investigación biomédica. Se trata de una RNA en la que las neuronas o nodos se organizan en capas, de modo que la salida de una neurona se dirige a todas y cada una de las neuronas de la capa siguiente. No existen conexiones laterales con neuronas de su misma capa, ni de capas precedentes. Las capas entre la primera y la última se consideran capas ocultas.

Cada entrada a una neurona es multiplicada por un peso, sumándose todas las entradas de un mismo nodo y añadiendo un término de sesgo. A esta suma ponderada se aplica más tarde una función de activación, que suele ser una función logística. En un primer momento los pesos y sesgos asociados a cada nodo se inicializan aleatoriamente, y durante el proceso de aprendizaje la red se aplica repetidamente a muestras de ensayo y la

Tabla 4

Valores de especificidad y puntos de corte para sensibilidades del 95\% y 90\% y áreas bajo la curva ROC

\begin{tabular}{|c|c|c|c|c|c|c|}
\hline & \multicolumn{2}{|c|}{ 95\% Sensibilidad } & \multicolumn{2}{|c|}{ 90\% Sensibilidad } & \multirow[t]{2}{*}{ ABC* } & \multirow[t]{2}{*}{$\mathbf{P}^{* *}$} \\
\hline & Punto de corte & Especificidad & Punto de corte & Especificidad & & \\
\hline $\mathrm{PSA}^{1}$ & $\geq 5,45$ & $7 \%$ & $\geq 6,15$ & $14,1 \%$ & 0,693 & 0,002 \\
\hline Volumen prostático ${ }^{2}$ & $\leq 102,75$ & $12,7 \%$ & $\leq 82,9$ & $25,4 \%$ & 0,707 & $<0,001$ \\
\hline Regresión logística & $\geq 0,3051$ & $35,2 \%$ & $\geq 0,4233$ & $60,6 \%$ & 0,815 & 0,760 \\
\hline Red neuronal artificial & $\geq 0,2648$ & $35,2 \%$ & $\geq 0,4104$ & $64,8 \%$ & 0,819 & \\
\hline
\end{tabular}

${ }^{1} \mathrm{ng} / \mathrm{ml}$.

${ }^{2}$ Centímetros cúbicos.

*Área bajo la curva ROC.

**Diferencias entre el ABC de PSA, volumen prostático y regresión logística, con respecto al ABC de la red neuronal (p calculada por procedimiento de DeLong).

ABC: Área bajo la curva 
salida de la red se compara con la salida deseada, midiendo la diferencia entre los valores obtenidos y los reales. Este proceso sirve como guía para variar los pesos y ajustarlos, de modo que los resultados producidos por la red se aproximen mejor a los buscados, hasta que finalmente el error total se haga menor que un nivel preestablecido $^{20}$.

Las RNA han sido utilizadas en diversos estudios para predecir el resultado de la BP, y aunque en todos ellos se muestran como un método eficaz, no siempre suponen una mejoría con respecto a los procedimientos estadísticos estándar. En este sentido, Sargent ${ }^{11}$ realiza una revisión de los principales estudios biomédicos en los que se compara la RNA con los métodos de regresión, tanto logística como de Cox, observando resultados heterogéneos, aunque en los estudios con mayor tamaño muestral, se observa una capacidad predictiva equivalente para ambos métodos.

Los estudios realizados por Djavan et al. ${ }^{21}$, Stephan et al. ${ }^{22}$ y Porter et $a .^{23}$, no encuentran diferencias entre la RNA y la regresión logística, en la predicción del resultado de la biopsia. Aún así, en los dos primeros estudios, las RNA demuestran una capacidad predictiva significativamente superior al PSA y sus formas moleculares.

Existen, por el contrario, otros estudios como los de Kalra et al. ${ }^{7}$ y Remzi et al. ${ }^{18}$, en los que la RNA se muestra significativamente superior a la regresión logística en la predicción de $\mathrm{CP}$ en la BP. El estudio de Remzi et al. ${ }^{18}$ está formado por pacientes con biopsia previa negativa y en él se calcula que el uso de la RNA permite reducir en un $68 \%$ el número de rebiopsias innecesarias.

En nuestro estudio hemos observado que la capacidad predictiva de la regresión logística $(\mathrm{ABC}$ : $0,815)$ y la red neuronal $(A B C: 0,819)$ son similares, y que cualquiera de las dos técnicas supera ampliamente la capacidad de los métodos diagnósticos unimodales en la predicción de $\mathrm{CP}$ en la $\mathrm{BP}$, ya que las ABC del PSA y del volumen prostático son de 0,693 y 0,707, respectivamente.

Hemos comprobado en nuestro análisis que la utilización de modelos predictivos basados en la combinación de variables, se asocia a un aumento importante de la especificidad en el diagnóstico del CP. Así, en nuestro estudio comprobamos que la especificidad del PSA para una sensibilidad del
95\% es tan sólo del 7\%. En el caso del volumen prostático, la especificidad es del 12,7\%. En el otro extremo observamos que la especificidad de la regresión logística y la red neuronal es del $35,2 \%$ en ambos casos, lo que quiere decir que ambos modelos permitirían ahorrar un $28 \%$ de biopsias innecesarias con respecto a la utilización aislada del PSA.

Si reducimos la sensibilidad de detección del $\mathrm{CP}$ a un $90 \%$, las diferencias observadas son aún mayores, ya que la especificidad del PSA sería de $14,1 \%$, la del modelo de regresión del $60,6 \%$ y la de la red neuronal del $64,8 \%$. Por lo tanto, la utilización de la red neuronal nos permitiría en este caso evitar más de un $50 \%$ de BP innecesarias.

El modelo de regresión logística y la RNA han sido confeccionados en nuestro estudio, utilizando una serie de variables claramente relacionadas con el CP, como son la edad, el tacto rectal y el PSA ${ }^{7}$. Además hemos añadido la variable volumen prostático, al observar en el análisis univariado una relación significativa con el resultado de la biopsia. Desde el punto de vista clínico el volumen prostático es un parámetro importante y para algunos autores, las variables relacionadas con el volumen prostático, ofrecen ventajas en sensibilidad y especificidad con respecto a otros parámetros $^{18,24}$.

La utilidad clínica de nuestro estudio y de otros similares consiste en que la RNA calcula una probabilidad individual de presentar CP en la biopsia. Esto permite, en primer lugar, individualizar la decisión de realizar una BP, en segundo lugar, superar la especificidad de los métodos diagnósticos unimodales, evitando biopsias innecesarias y en tercer lugar, al asignar a cada paciente una probabilidad de $\mathrm{CP}$, determinar en qué casos resulta necesario repetir la BP, cuando la primera biopsia es negativa ${ }^{18,21}$.

En el presente trabajo hemos testado una red neuronal, comparándola con un modelo de regresión logística, basados ambos en las mismas variables clínicas. Los resultados observados sugieren que las redes neuronales constituyen una herramienta alternativa en el diagnóstico del cáncer de próstata, aunque no mejoran significativamente los resultados obtenidos con las técnicas estadísticas habituales. Esta conclusión debería 
ser refrendada posteriormente con un volumen muestral mayor. Sería también interesante desarrollar una RNA seleccionando grupos de pacientes con cifras de PSA en los intervalos donde este parámetro presenta una menor especificidad, incluyendo asimismo en el análisis variables relacionadas con el PSA como cociente PSA libre/total y PSA densidad, entre otras.

\section{REFERENCIAS}

1. Allepuz Losa C, Borque Fernando Á, Rioja Sanz LA. ¿Cuál es el algoritmo diagnóstico más apropiado para el cribado del cáncer de próstata? Actas Urol Esp 2005;29(5):465-472.

2. Catalona WJ, Southwick PC, Slawin KM, Partin AW, Brawer MK, Flanigan RC, et al. Comparison of percent free PSA, PSA density, and age-specific PSA cutoffs for prostate cancer detection and staging. Urology 2000;56(2):255-260.

3. Montie JE, Meyers SE. Defining the ideal tumor marker for prostate cancer. Urol Clin North Am 1997;24(2):247-252.

4. Partin AW, Oesterling JE. The clinical usefulness of prostate specific antigen: Update 1994. J Urol 1994;152:13581368.

5. Arcangeli CG, Ornstein DK, Keetch DW, Andriole GL. Prostate-specific antigen as a screening test for prostate cancer. The United States experience. Urol Clin N Am 1997;24(2):299-306.

6. Catalona WJ, Partin AW, Slawin KM, Brawer MK, Flanigan $\mathrm{RC}$, Patel A, et al. Use of the percentage of free prostatespecific antigen to enhance differentiation of prostate cancer from benign prostatic disease: a prospective multicenter clinical trial. JAMA 1998;279:1542-1547.

7. Kalra P, Togami J, Bansal G, Partin AW, Brawer MK, Babaian RJ, et al. A neurocomputational model for prostate carcinoma detection. Cancer 2003;98(9):1849-1854.

8. Morote J, Ruibal A. El antígeno prostático específico. Nuevo marcador tumoral. Med Clin 1985;85:801-804.

9. Wei JT, Zhang Z, Barnhill SD, Madyastha R, Zhang H, Oesterling JE, et al. Understanding artificial neural networks and exploring their potential applications for the practicing urologist. Urology 1998;52(2):161-172.

10. Dayhoff JE, DeLeo JM. Artificial neural networks. Opening the black box. Cancer 2001;91(8)Suppl.1:615-1635.

11. Sargent DJ. Comparison of artificial neural networks with other statistical approaches. Cancer 2001;91(8)Suppl.1: 1636-1642.

12. Borque A, Sanz G, Allepuz C, Plaza L, Gil P, Rioja LA. The use of neural networks and logistic regresión análisis for predicting pathological stage in men undergoing radical prostatectomy: a population based study. J Urol 2001; 166:1672-1678.

13. Han M, Snow PB, Brandt JM, Partin AW. Evalutation of artificial neural networks for the prediction of pathologic stage in prostate carcinoma. Cancer 2001;91(8)Suppl.1: 1661-1666.
14. Reckwitz T, Potter SR, Snow PB, Zhang Z, Veltri RW, Partin AW. Artificial neural networks in urology: update 2000. Prostate Cancer Prostatic Dis 1999;2:222-226.

15. Littrup PJ, Williams CR, Egglin TK, Kane RA. Determination of prostate volume with transrectal US for cancer screening. Part II. Accuracy of in vitro and in vivo techniques. Radiology 1991;179:49. (Completar referencia en medline).

16. DeLong ER, DeLong DM, Clarke-Pearson DL. Comparing the areas under two or more correlated receiver operating curves: a non parametric approach. Biometrics 1988;44: 837-845.

17. Bangma CH, Rietbergen JB, Schröder FH. Prostate-specific antigen as a screening test. The Netherlands experience. Urol Clin North Am 1997;24(2):307-314.

18. Remzi M, Anagnostou T, Ravery V, Zlotta A, Stephan C, Marberger M, et al. An artificial neural network to predict the outcome of repeat prostate biopsies. Urology 2003; 62(3):456-460.

19. Levine RF. Clinical problems, computational solutions. A vision for a collaborative future. Cancer 2001;91(8) Suppl.1:1595-1602.

20. Hinton GE. How neural networks learn from experience. Sci Am 1992;267:144-151.

21. Djavan B, Remzi M, Zlotta A, Seitz C, Snow P, Marberger M. Novel artificial neural network for early detection of prostate cancer. J Clin Oncol 2002;20(4):921-929.

22. Stephan C, Cammann H, Semjonow A, Diamandis P, Wymenga LFA, Lein M, et al. Multicenter evaluation of an artificial neural network to increase the prostate cancer detection rate and reduce unnecesary biopsies. Clin Chem 2002;48(8):1279-1287.

23. Porter CR, Gamito EJ, Crawford D, Bartsch G, Presti JC, Tewari A, et al. Model to predict prostate biopsy outcome in large screening population with independent validation in referral setting. Urology 2005;65(5):937-941.

24. Djavan B, Remzi M, Zlotta AR, Ravery V, Hammerer P, Reissigl A, et al. Complexed prostate-specific antigen, complexed prostate-specific antigen density of total and transition zone, complexed/total prostate-specific antigen ratio, free-to-total prostate-specific antigen ratio, density of total and transition zone prostate-specific antigen: Results of the Prospective Multicenter European Trial. Urology 2002;60Suppl.4A:4-9.

Dr. A. Rodríguez Alonso

Servicio de Urología

Hospital Arquitecto Marcide-Profesor Novoa Santos Ctra. San Pedro de Leixa s/n

15405, Ferrol, La Coruña

E-mail: arodri68@terra.es

(Trabajo recibido el 21 de septiembre 2005) 